\title{
A Case of Bilateral Secondary Pneumothorax Shortly after the Completion of Concurrent Chemoradiotherapy for Tongue Cancer
}

\author{
Yoshiro Saito1,2*, Hideyuki Katsuta1,2,3, Takashi Moriya1,2, Yuya Kurasawa1,2, Hitoshi Sato1,2, \\ Shunya Egawa1,2,3, Yukiomi Kushihashi1,2,3, Kenichiro Ikeda 1,2,3, Toshikazu Shimane 1,2,3 \\ ${ }^{1}$ Head and Neck Oncology Center, Showa University, Tokyo, Japan \\ ${ }^{2}$ Department of Oral and Maxillofacial Surgery, Division of Oral Oncology, Showa University, School of Dentistry, Tokyo, Japan \\ ${ }^{3}$ Department of Otorhinolaryngology, Showa University School of Medicine, Tokyo, Japan \\ Email: *y.saito62@dent.showa-u.ac.jp
}

How to cite this paper: Saito, Y., Katsuta, H., Moriya, T., Kurasawa, Y., Sato, H., Egawa, S., Kushihashi, Y., Ikeda, K. and Shimane, T. (2020) A Case of Bilateral Secondary Pneumothorax Shortly after the Completion of Concurrent Chemoradiotherapy for Tongue Cancer. International Journal of Otolaryngology and Head \& Neck Surgery, 9, 93-100.

https://doi.org/10.4236/ijohns.2020.93013

Received: April 7, 2020

Accepted: May 18, 2020

Published: May 21, 2020

Copyright ( 2020 by author(s) and Scientific Research Publishing Inc. This work is licensed under the Creative Commons Attribution International License (CC BY 4.0).

http://creativecommons.org/licenses/by/4.0/

\section{(c) (i) Open Access}

\begin{abstract}
Metastatic lung tumours rarely lead to development of pneumothorax, and no case of bilateral secondary pneumothorax due to lung metastases arising from tongue cancer has been reported. Here, we report a case of a patient with tongue cancer with lung metastases complicated by bilateral secondary pneumothorax soon after the completion of concurrent chemoradiotherapy. A 39-year-old man with cervical lymph node metastases originating from pT2N0M0 tongue cancer underwent neck dissection and postoperative concurrent chemoradiotherapy. Shortly after the completion of chemoradiotherapy, he developed bilateral secondary pneumothorax. Subsequently, he underwent partial lung resection for the pulmonary fistulae for diagnostic and therapeutic purposes; nodular lesions found in both the lungs. The diagnosis of secondary pneumothorax was based on histopathological findings. Although all pulmonary fistulae disappeared after partial lung resection, he died of the primary disease despite our best efforts to control the metastatic pulmonary lesions.
\end{abstract}

\section{Keywords}

Tongue Cancer, Metastatic Lung Tumour, Pneumothorax, Concurrent Chemoradiotherapy

\section{Introduction}

According to reports, metastatic lung tumour rarely causes pneumothorax and the prevalence of metastatic lung tumour leading to pneumothorax is less than 
$1 \%$ [1]. Despite the fact that the most common site of distant metastasis in tongue cancer is the lung, only a few studies have reported secondary pneumothorax caused by lung metastasis from tongue cancer [2]. In this case study, we have reported the case of patient with late cervical lymph node metastases from tongue cancer (pT2N0M0), who was diagnosed with bilateral pneumothorax after undergoing neck dissection and postoperative concurrent chemoradiotherapy (CCRT). Although this occurs very rarely, to maintain the patient's QoL, lung metastasis should be closely watched for any complication.

\section{Case Presentation}

A 39-year-old man presented to our hospital with the chief complaint of an ulcer on the right lateral border of the tongue. He had a history of myelodysplastic syndrome and received cord blood transplantation for the treatment of the syndrome. Subsequently, he was diagnosed with chronic graft versus host disease (chronic GVHD) and received steroid therapy. A $21 \times 16 \mathrm{~mm}$ tongue tumour was observed on the right lateral border of the tongue (Figure 1). Based on the contrast-enhanced MRI T1-weighted images, the invasion depth was $6 \mathrm{~mm}$ (Figure 2). Contrast-enhanced CT displayed no cervical lymph node metastasis or distant metastasis including lung metastasis. He was diagnosed with right tongue cancer (cT2N0M0) and underwent partial resection of the right tongue. Histopathological examination showed invasive growth of atypical squamous cells with enlarged nucleoli and alveolar cancer cells, along with cancer pearls with keratinization in the centre of the nest (Figure 3). Therefore, the diagnosis was confirmed as squamous carcinoma in right tongue pT2N0M0 (stage II). Subsequently, right neck dissection (levels I to V) were performed because late cervical lymph node metastases were observed in the right submandibular region and the mid-internal jugular region. Histopathological findings revealed metastatic lymph nodes in the submandibular (5/11), mid-internal jugular (10/31), and lower-internal jugular region (2/10). The total number of metastatic lymph nodes was 17/67 of which, 12 lymph nodes showed extra-nodal invasion. Postoperative contrast-enhanced CT images demonstrated no lung metastasis. Subsequently, he was diagnosed with multiple lymph node metastases and extra-nodal tumour invasion and received CCRT (three courses of cisplatin 80 $\mathrm{mg} / \mathrm{m}^{2}$; total of $420 \mathrm{mg} /$ body; total of $66 \mathrm{~Gy} / 33 \mathrm{fr}$ ) as postoperative adjuvant therapy. Shortly after the completion of CCRT, he made an emergency visit to our centre with complaints of respiratory discomfort and chest pain. Chest $\mathrm{x}$-ray showed complete collapse of bilateral lung fields, and he was diagnosed with bilateral pneumothorax (Figure 4). Chest CT showed nodules and cavities in both the lung fields (Figure 5). Therefore, primary or secondary pneumothorax due to lung metastases from tongue cancer was considered. However, even after subsequent bilateral thoracic drainage, there was persistence of pulmonary fistulae. Since conservative treatment was imvalid, we made a plan to bilateral thoracoscopic partial resection of the lungs for diagnostic and therapeutic purposes. 


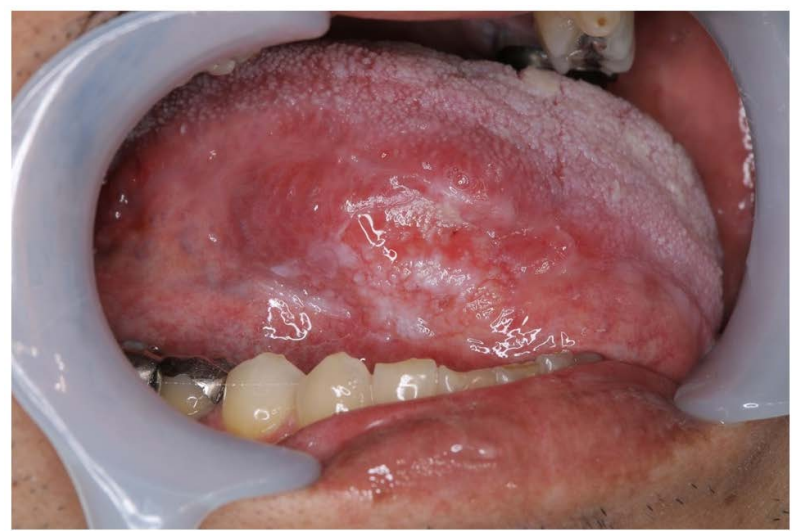

Figure 1. Intraoral findings at the first visit. A $21 \times 16 \mathrm{~mm}$ tumour with induration was observed on the right lateral border of the tongue.

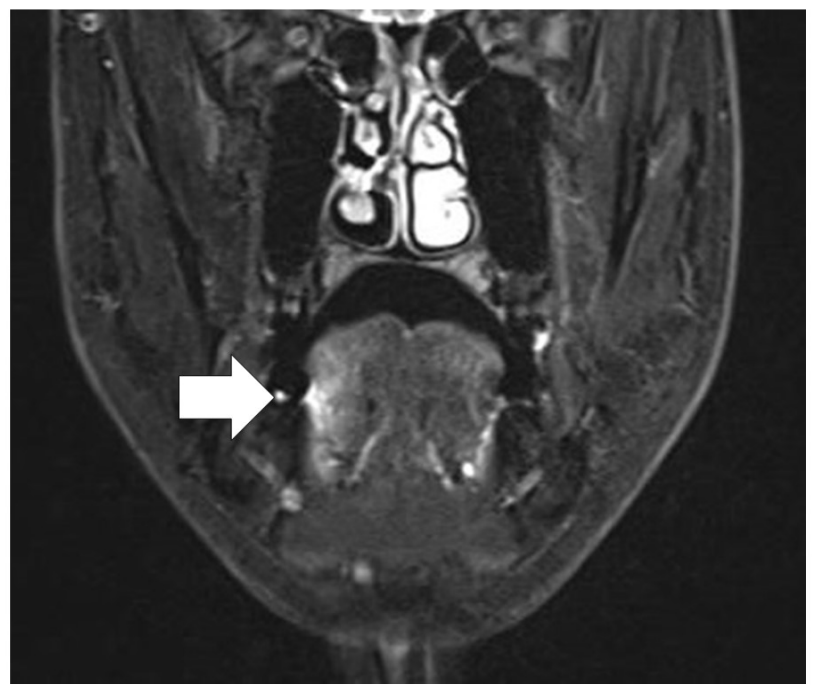

Figure 2. Contrast-enhanced MRI T1-weighted image. A contrast-enhanced MRI T1-weighted image showed a high intensity area on the right lateral border of the tongue (white arrow).
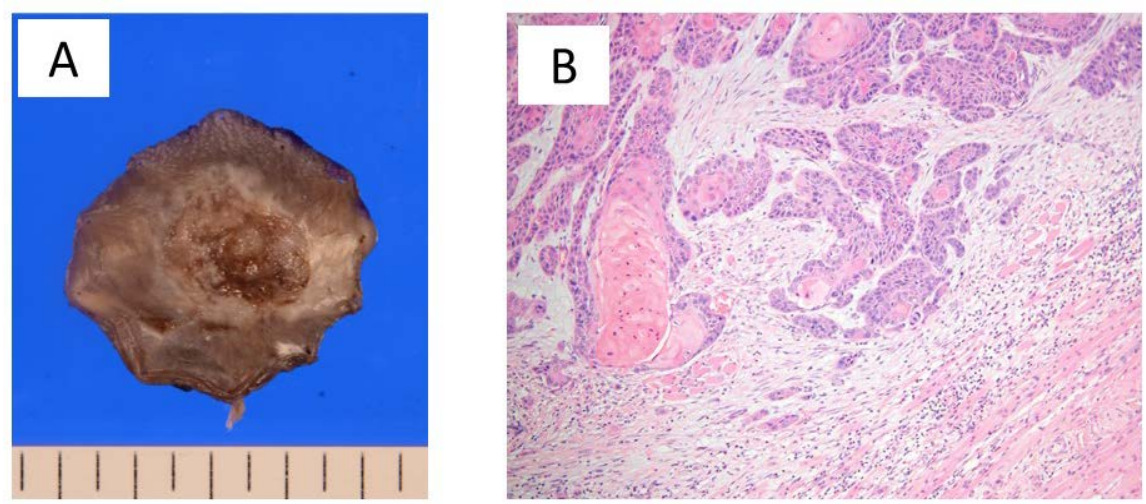

Figure 3. (A) Macroscopic image of the tongue cancer. (B) Histopathological image of the tissue derived from partial glossectomy $(\mathrm{H}-\mathrm{E}$ staining, $\times 200)$. Growing alveolar cancer cells and cancer pearls with keratinization in the centre of the nests were observed. The patient was diagnosed with well-differentiated squamous cell carcinoma. 


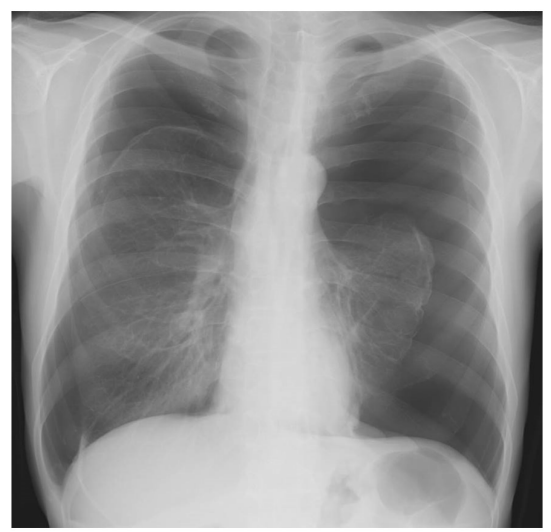

Figure 4. Chest X-ray image. Complete collapse of both lung fields was observed.
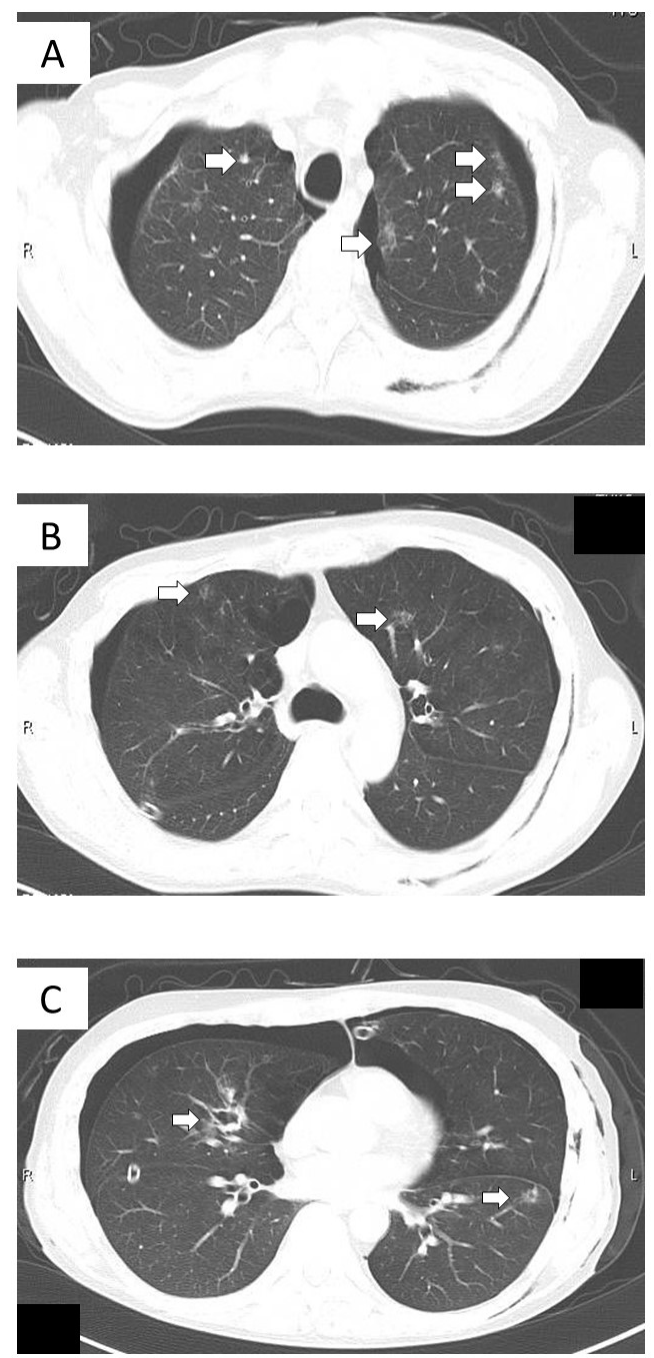

Figure 5. Simple chest CT images at the onset of pneumothorax. Numerous nodules and cavities were observed in both lungs (White arrows). (A) Cross-sectional image of the upper mediastinum. Lesions were observed in the right S3 and left S3 regions; (B) Cross-sectional image of the aortic arch. Lesions were observed in the right S3 and left S3 regions; (C) Cross-sectional image of the aortic arch. Lesions were observed in the right S4 and left S9 regions. A pulmonary fistula was observed in the right S4 region. 
Partial resection of nodular lesions in the S1 and S2 regions of the left lung along with closure of pulmonary fistulae in the S6 region was performed. Additionally, a partial resection of nodular lesions and pulmonary fistulae in the S3 and S4 regions of the right lung was performed. Finally, pleurodesis was undertaken to prevent the recurrence of pneumothorax. Histopathological findings suggested poorly differentiated squamous cell carcinoma with proliferation of atypical cells containing nuclei with dark chromatin in both lungs. The final diagnosis was lung metastases from tongue cancer based on the above histopathological and clinical findings. Based on pleural tumour invasion and the formation of pulmonary embolism, he was diagnosed with secondary pneumothorax due to lung metastases from tongue cancer (Figure 6). Despite the postoperative closure of the pulmonary fistulae and a temporary improvement in the respiratory status, the patient died due to the primary disease after losing control of lung metastatic lesions.
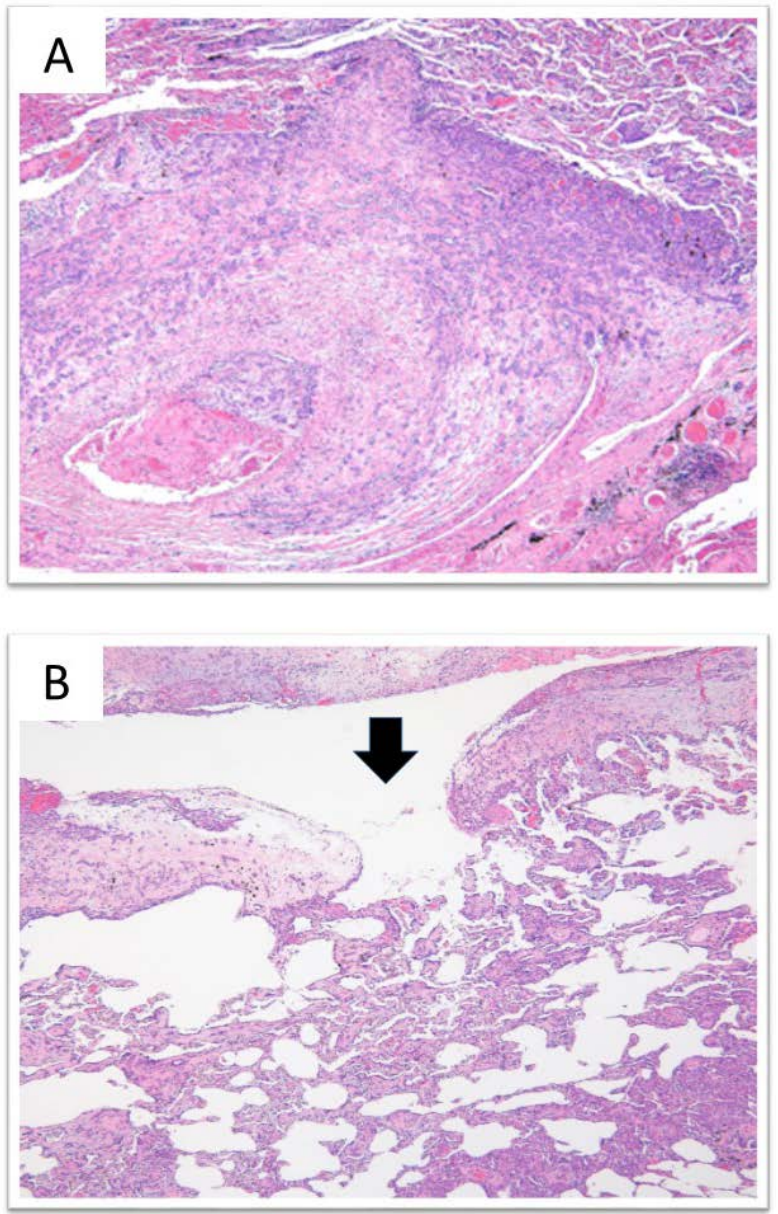

Figure 6. Histopathological findings of the tissue derived from partial lung resection. The proliferation of cancer cells possessing nuclei with increased chromatin was observed, and the patient was diagnosed with poorly differentiated squamous cell carcinoma. (A) Partial resection of the left lung (H-E staining, $\times 200$ ); (B) Partial resection of the right lung (H-E staining, $\times 200)$. Black arrow: Pleural invasion of cancer cells and damage to the pleura. 


\section{Discussion}

The rate of distant metastasis in head and neck cancer with primary tumours and cervical metastases controlled has been reported to be $7.0 \%-23 \%$ [3]-[9]. In particular, the prevalence of distant metastases arising from oral cancer is low (7\%) [5] [7] [10]. Lung is the most common site of distant metastases from oral squamous cell carcinomas including tongue cancer. Furthermore, it is difficult to rapidly treat multiple distant metastases in head and neck squamous cell carcinomas because this may lead to worsening of the general condition of the patient.

In the absence of any coexisting pathology, spontaneous pneumothorax is considered a primary pneumothorax. However, if associated with an underlying lung disease, it is considered a secondary pneumothorax. Primary lung cancer and metastatic lung tumours can both cause secondary pneumothorax, although it is rarely seen with metastatic lung tumours with a prevalence of $<1 \%$ [1]. In majority of the cases of pneumothorax, the metastatic lung tumour originates from a sarcoma [11]. There are only a few reports of pneumothorax attributed to lung metastases arising from an oral cancer. The possible mechanisms for development of pneumothorax due to metastatic lung cancer [2] are as follows: 1) formation of a bronchopleural fistula due to necrosis of subpleural metastases (necrosis, treatment, and infection); 2) compression due to metastases or invasion of bronchiolar tumours followed by the rupture of a cyst (formed possibly because of a check-valve mechanism) with necrotic substance discharged into the bronchial lumen; 3) positive pressure in the tumour cavity because of a check-valve mechanism due to tumour centre necrosis; and 4) direct tumour metastasis and invasion of the existing pulmonary cyst wall and pleura. In this study, the histopathological findings of the fistula from the right pulmonary artery showed pleural invasion by the squamous cell carcinoma. Therefore, the patient was diagnosed with pneumothorax based on 4). The patient developed pneumothorax shortly after the completion of chemotherapy. This suggests that the pneumothorax developed because of tumour necrosis of the lung metastatic lesions as a result of the antitumour effects of the anticancer drugs. Felon HM et al. [12] reported a similar case with a bilateral pneumothorax formation following chemotherapy for a sarcoma. Multiple pathogenetic mechanisms could have been responsible for the development of pneumothorax in the present case.

It is essential to consider the treatment strategy for malignant tumours and patient quality of life (QoL) prior to management of pneumothorax. In majority of the cases, the cancer is in the advanced stages at the onset of the pneumothorax. Therefore, complications such as respiratory failure may lead to the worsening of the general condition of the patient. It is important to keep in mind that chemotherapy in patients with suspected lung metastasis from tongue cancer may lead to secondary pneumothorax. Although this occurs very rarely, to maintain the patient's QoL, lung metastasis should be closely watched for any complication. 


\section{Conclusion}

We report a case of a patient with tongue cancer with lung metastases complicated by bilateral secondary pneumothorax soon after the completion of concurrent chemoradiotherapy.

\section{Conflicts of Interest}

The authors declare no conflicts of interest regarding the publication of this paper.

\section{References}

[1] Dines, D., Cortese, D., Brennan, M.D., Hahn, R.G. and Payne, W.S. (1973) Malignant Pulmonary Neoplasms Predisposing to Spontaneous Pneumothorax. Mayo Clinic Proceedings, 48, 541-544.

[2] Hsu, J.S., Chou, S.H., Tsai, K.B. and Chuang, M.T. (2009) Lingual Carcinoma Metastases Presenting as Spontaneous Pneumothorax. Journal of the Formosan Medical Association, 108, 736-738. https://doi.org/10.1016/S0929-6646(09)60398-3

[3] Ellis, E.R., Mendenhall, W.M., Rao, P.V., Parsons, J.T., Spangler, A.E. and Million, R.R. (1989) Does Node Location Affect the Incidence of Distant Metastases in Head and Neck Squamous Cell Carcinoma? International Journal of Radiation Oncology, Biology, Physics, 17, 293-297. https://doi.org/10.1016/0360-3016(89)90442-2

[4] Nishijima, W., Takooda, S., Tokita, N., Takayama, S. and Sakura, M. (1993) Analyses of Distant Metastases in Squamous Cell Carcinoma of the Head and Neck and Lesions above the Clavicle at Autopsy. Archives of Otolaryngology-Head and Neck Surgery, 119, 65-68. https://doi.org/10.1001/archotol.1993.01880130067009

[5] Leibel, S.A., Scott, C.B., Mohiuddin, M., Marcial, V.A., Coia, L.R., et al. (1991) The Effect of Local-Regional Control on Distant Metastatic Dissemination in Carcinoma of the Head and Neck: Results of an Analysis from the RTOG Head and Neck Database. International Journal of Radiation Oncology, Biology, Physics, 21, 549-556. https://doi.org/10.1016/0360-3016(91)90669-U

[6] Leemans, C.R., Tiwari, R., Nauta, J.P.J., Van der Waal, I. and Snow, G.B. (1993) Regional Lymph Node Involvement and Its Significance in the Development of Distant Metastases in Head and Neck Carcinoma. Cancer, 71, 452-456. https://doi.org/10.1002/1097-0142(19930115)71:2<452::AID-CNCR2820710228>3.0 .CO;2-B

[7] Papac, R.J. (1984) Distant Metastases from Head and Neck Cancer. Cancer, 53, 342-345.

https://doi.org/10.1002/1097-0142(19840115)53:2<342::AID-CNCR2820530228>3.0 .CO;2-9

[8] Shintani, S., Matsuura, H., Hasegawa, Y., Nakayama, B. and Hasegawa, H. (1995) Regional Lymph Node Involvement Affects the Incidence of Distant Metastasis in Tongue Squamous Cell Carcinomas. Anticancer Research, 15, 1573-1576.

[9] Shingaki, S., Suzuki, I., Kobayashi, T. and Nakajima, T. (1996) Predicting Factors for Distant Metastases in Head and Neck Carcinomas: An Analysis of 103 Patients with Locoregional Control. Journal of Oral and Maxillofacial Surgery, 54, 853-857. https://doi.org/10.1016/S0278-2391(96)90535-2

[10] Alvi, A. and Johnson, J.T. (1997) Development of Distant Metastasis after Treatment of Advanced-Stage Head and Neck Cancer. Head \& Neck, 19, 500-505. 
https://doi.org/10.1002/(SICI)1097-0347(199709)19:6<500::AID-HED7>3.0.CO;2-2

[11] Smevik, B. and Klepp, O. (1982) The Risk of Spontaneous Pneumothorax in Patients with Osteogenic Sarcoma and Testicular Cancer. Cancer, 49, 1734-1737.

https://doi.org/10.1002/1097-0142(19820415)49:8<1734::AID-CNCR2820490833>3. $0 . \mathrm{CO} ; 2-\mathrm{K}$

[12] Fenlon, H.M., Carney, D. and Breatnach, E. (1996) Case Report: Bilateral Recurrent Tension Pneumothorax Complicating Combination Chemotherapy for Soft Tissue Sarcoma. Brain and Language, 51, 302-304.

https://doi.org/10.1016/S0009-9260(96)80353-9 